\title{
Reviewers of BIBECHANA- 2018
}

PROF. IOSIF GALANAKIS

PROF. RAJU KHANAL

PROF. NARAYAN PRASAD ADHIKARI

Kul PRASAD LIMBU

PROF. A. WEISS

DR. DIL KUMAR LIMBU

DR. GULSHAN SINGH

PROF D. ADHIKARI

DR. CUT TIAN

PROF. O. C. JONES

PROF. XUEFANG DAI

PROF R.N. JORDAR

PROF. B.P. SINGH

PROF. P.R. PRADHAN

DR. ANANT BABU MaRAHATtA

DR. J. KUMAR

PROF. B.K. SINGH 